\title{
Potential barriers to rapid testing for human immunodeficiency virus among a commuter population in Johannesburg, South Africa
}

This article was published in the following Dove Press journal:

HIVIAIDS - Research and Palliative Care

30 December 2014

Number of times this article has been viewed

\author{
Ndumiso Tshuma' \\ Keith Muloongo' \\ Geoffrey Setswe ${ }^{2}$ \\ Lucy Chimoyi ${ }^{4}$ \\ Bismark Sarfo ${ }^{5}$ \\ Dina Burger 6 \\ Peter S Nyasulu ${ }^{3,7}$ \\ 'Community AIDS Response, \\ Norwood, Johannesburg, South \\ Africa; ${ }^{2} \mathrm{HIV} / \mathrm{AIDS}, \mathrm{STI}$ and TB \\ (HAST) research program, Human \\ Science Research Council (HSRC), \\ Pretoria, South Africa; ${ }^{3}$ School of \\ Public Health, University of the \\ Witwatersrand, Johannesburg, South \\ Africa; ${ }^{4}$ Wits Reproductive Health \\ and HIV Research Institute (WHRI), \\ Hillbrow, Johannesburg, South Africa \\ ${ }^{5}$ Department of Epidemiology and \\ Disease Control, School of Public \\ Health University of Ghana, Legon- \\ Accra, Ghana; ${ }^{6}$ The Research Office, \\ Monash South Africa, Ruimsig, \\ Johannesburg, South Africa; ${ }^{7}$ School of \\ Health Sciences, Monash University, \\ Ruimsig, Johannesburg, South Africa
}

Correspondence: Peter S Nyasulu School of Health Sciences,

Monash University, I44 Peter Road, Ruimsig, Johannesburg, South Africa Tel +27 I | 9504287

Email peter.nyasulu@monash.edu
Background: This study aimed to determine barriers to accessing human immunodeficiency virus (HIV) counseling and testing (HCT) services among a commuter population.

Methods: A cross-sectional, venue-based intercept survey was conducted. Participants were recruited during a 2-day community campaign at the Noord Street taxi rank in Johannesburg, South Africa. Data were collected using a self-administered questionnaire loaded onto an electronic data collection system and analyzed using Stata software. Factors contributing to barriers for HCT were modeled using multivariate logistic regression.

Results: A total of 1,146 (567 male and 579 female) individuals were interviewed; of these, $51.4 \%$ were females. The majority $(59.5 \%)$ were aged $25-35$ years. Significant factors were age group (15-19 years), marital status (married), educational level (high school), distance to the nearest clinic $(>30 \mathrm{~km})$, area of employment/residence (outside inner city), and number of sexual partners (more than one). Participants aged 15-19 years were more likely to report low-risk perception of HIV as a barrier to HCT (odds ratio [OR] 1.62; 95\% confidence interval [CI] 1.01-2.59), the married were more likely to report low-risk perception of HIV as a barrier to HCT (OR 1.49; 95\% CI 1.13-1.96), and those living outside the inner city were more likely to report lack of partner support as a potential barrier (OR 1.94; 95\% CI 1.34-2.80), while those with a high school education were more likely to report poor health worker attitude as a potential barrier to HIV testing (OR 2.17; 95\% CI 1.36-3.45).

Conclusion: Age, marital status, occupation, educational level, area of employment and residence, distance to the nearest clinic, and number of sexual partners were factors significantly associated with barriers to HIV testing in the study population. Future HIV intervention targeting this population need to be reinforced in order to enhance HIV testing while taking cognizance of these factors.

Keywords: human immunodeficiency virus, testing, counseling, commuter, population, accessibility

\section{Introduction}

South Africa has the highest number of people living with human immunodeficiency virus (HIV) in the world. Over 10\% of the population (5.6 million) is infected, of which an estimated $15.9 \%$ of the productive age group of $15-49$ years are HIV-positive. ${ }^{1}$ An effective national strategy to control HIV infection would require expanded access to HIV counseling and testing (HCT). The approach of using stationary HCT health care facilities alone will not result in the achievement of national HIV prevention targets. ${ }^{2}$ In response, several nongovernmental organizations, including Community AIDS Response (CARe), have been working to support government initiatives to increase access to HCT services. This is done through the implementation of home-based and 
mobile focused $\mathrm{HCT}^{3}$ Despite efforts to increase the uptake of HIV testing, there are still barriers to testing among urban communities. ${ }^{4,5}$ The South African National HIV Prevalence, Incidence, Behaviour and Communication Survey reported that Gauteng province has the lowest HIV testing participation rate in the country, with only $59.4 \%$ of individuals agreeing to HIV testing. The highest testing response rate in urban informal settlements was $72.5 \%$ and the lowest in the rural formal areas was $72.6 \%$, while urban formal areas recorded $62.8 \%{ }^{6}$

HCT services are a vehicle through which millions of people learn about their HIV status. For individuals who test positive, HCT provides a platform for them to select health care services that provide opportunities for longterm care and treatment. ${ }^{7}$ It is widely acknowledged that testing for HIV decreases the likelihood of high-risk behavior among individuals who choose to test, which leads to reduction in HIV transmission. ${ }^{8}$ For this reason, there is a drive to increase the prevalence and accessibility to HCT. In response to the country's growing demand for HIV testing, the South African Department of Health launched a nationwide HIV counseling and testing campaign in 2010 to increase testing rates and provide a stepping stone to antiretroviral therapy. ${ }^{9}$ The campaign aimed at reducing the incidence of HIV infection by $50 \%$; however, it was not successful in meeting its goal by the projected deadline set for June 2011. ${ }^{10}$

Structural and cultural barriers in South Africa threaten to undermine HCT campaigns when they are not addressed. There have been several studies within the South African context that measure barriers to HCT. For example, in 2005, a study of the effectiveness of a mother to child transmission prevention program found that structural factors such as weak management capacities in health centers contributed to lower rates of HCT. Clinics with stronger management capacities were able to employ lay counselors to lessen the burden on nurse counselors, integrate policy into practice, secure test kit supplies, and increase the rate of HCT uptake. The authors concluded that a strong management capacity is essential to the successful implementation of HCT programs. ${ }^{11}$

Many of the studies performed on barriers to testing in South Africa have targeted townships as study sites and have usually targeted testing cultural barriers, primarily focusing on HIV-related knowledge, risk perception, and HIV/acquired immune deficiency syndrome (AIDS)-related stigma. In 2003, a study performed in a black township in Cape Town found that HIV-related knowledge is not associated with testing history, which underscores findings by other authors who suggested that HIV-related knowledge was necessary in increasing HIV testing rates, but was not sufficient when other barriers outweighed the potential benefits of testing. ${ }^{12}$ HIV/AIDS-related stigma has been cited as a barrier to HIV testing despite the presence of knowledge about HIV transmission. ${ }^{12}$ Shisana et al found that HIV/AIDS-related stigma in South Africa has actually been decreasing since 2002. However, a recent study has demonstrated that HIVrelated stigma is a prominent barrier to testing. ${ }^{10}$ Studies also identify a low perceived risk as a problematic barrier to HIV testing. Individuals who perceived their risk of HIV infection as low are likely to engage in high-risk behaviors, and do not feel the need for HIV testing. However, an individual's self-perceived risk may often be inaccurate. ${ }^{13}$ Furthermore, a lower risk perception correlates with higher rates of HIV infection. $^{14}$

Barriers to testing delay the entry of HIV-infected individuals to the continuum of care, despite sufficient evidence supporting the value of seeking HCT services. This leads to delayed diagnosis of HIV infection, increased morbidity, increased health care costs, and ultimately high mortality of those infected. ${ }^{15}$ Commuter populations, such as those found commuting through urban centers, often have access to higher concentrations of social amenities, such as health care services. However, individuals in the inner city are faced with challenges of high population density and cultural diversity, compounding the barriers to testing. For example, the Johannesburg metropolitan population is comprised of a very culturally diverse populace, with Gauteng absorbing more immigrants with diverse cultural backgrounds than any other South African province. ${ }^{16}$ Therefore, trying to provide health care services to individuals from such a wide range of cultural backgrounds could present additional and unique challenges to HCT uptake. ${ }^{17}$

The levels of access to health care, population density, and cultural diversity of Johannesburg's inner city combine to form a unique environment and valuable location for study of the barriers to HCT for commuter populations. Since data on barriers to HCT uptake in this population is limited, conducting such a study would provide a platform for understanding the risk behaviors and determinants as well as sociodemographic factors associated with HIV testing in this special population and indeed in the developing world's mobile populations who are at higher risk of HIV infection. ${ }^{18}$ Studies on barriers to HCT in South Africa have traditionally focused on individuals moving through the formal health 
care system, ${ }^{11,19}$ individuals living in townships, ${ }^{10,12,13}$ or the general population. ${ }^{14}$

This study focused on commuter populations, who are groups of people who travel some distance to and from work or in search of work on a regular basis. ${ }^{20}$ The study aimed at increasing knowledge and understanding of barriers to accessing HCT services within the commuter population in order to inform policy decisions and intervention strategies at the district levels targeting commuter populations to enhance uptake of HCT services.

\section{Materials and methods Study design}

The study used a simple random sampling technique to select participants for a venue-based intercept survey at the four corners of Noord Street taxi rank in the Johannesburg inner city central business district (CBD). The simple random sampling technique entailed selection of an even number from a bowl with numbers 1 to 10 . Field workers approached potential participants walking toward the corners that served as intercept points and sought verbal consent prior to allowing potential participants to pick the numbers from a bowl. Only those who picked an even number were allowed to respond to a closed-ended questionnaire. Field workers were on standby to provide support to participants who had any challenges in answering the questions. The participants were also offered free voluntary HCT after completion of the questionnaire. However, acceptance levels were not tracked.

\section{Study setting}

The study was conducted at the Noord Street taxi rank in Johannesburg, South Africa (Figure 1). The Noord Street taxi
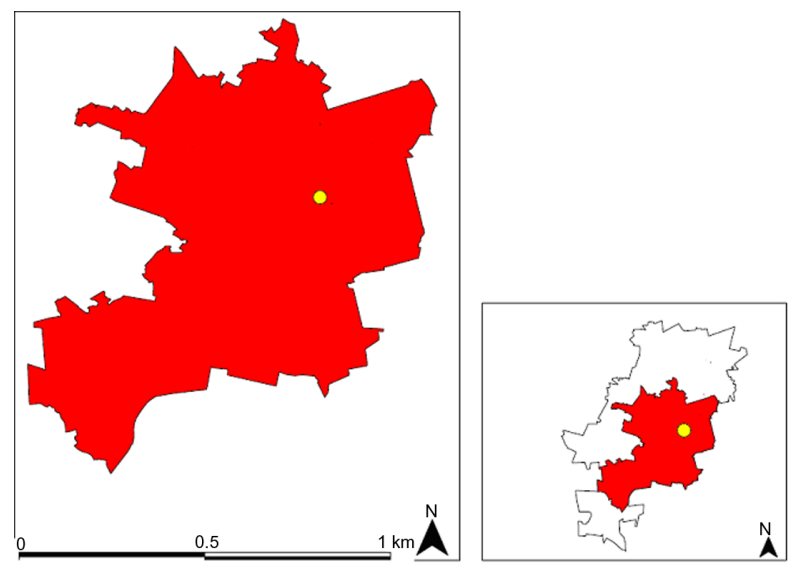

Figure I Map of Johannesburg (white) showing the position of the inner city (red) and the Noord Taxi rank (yellow) where the study was conducted. rank is by far the largest and busiest taxi rank in Johannesburg and is positioned at the heart of the inner city in the CBD. ${ }^{21}$ During apartheid, the CBD was Johannesburg's commercial center, home to many of the city's national and international companies. However, following the dismantling of apartheid, the CBD made a swift and disorderly transition, absorbing many of the urban poor, while a majority of businesses and corporations fled to upper class suburbs, such as Sandton.

Individuals came to the inner city from townships that were inconveniently far from business opportunities, for cheaper transportation costs, job opportunities, and more convenient access to social amenities. The CBD also saw a large influx of migrants seeking job opportunities in Johannesburg, both from other provinces and from neighboring countries. $^{22}$ The 2011 census reported that Gauteng absorbed a net migration of 1,037,871 individuals between 2001 and 2011, being the highest in the country. ${ }^{16}$ As a result, the CBD has transformed from a spacious, well-maintained business district to a densely populated, culturally diverse, and poorly regulated city center over a short period of time. ${ }^{22}$

The Noord Street taxi rank is Johannesburg's primary transit center for all mini taxis operating in South Africa's mini-taxi system. It is common knowledge that taking a minitaxi is often inconvenient and time-consuming, but is also the cheapest means of transport from one part of the city to another. Therefore, the mini-taxi system is primarily utilized by individuals without the means to afford more comfortable and convenient modes of transport, such as private cars or metered taxis. Most passengers use the Noord taxi rank as a platform to start their commute or switch directions and travel to a different area of the city. Individuals moving through the Noord taxi rank come not only from townships at the city periphery, but also from the CBD itself. The CBD has high levels of unemployment and is adjusting to relatively newly increased population densities. Often, the only tangible housing options for individuals living in the CBD are illegal, informal renting spaces, which tend to operate out of dilapidated buildings. It is estimated that 100,000 of the city's residents utilize informal rental housing in the inner city. ${ }^{22}$

\section{Data collection}

Data were collected via a self-administered closed-ended questionnaire loaded onto an electronic data collection system using personal tablet computers. Data were collected during a 2-day campaign at the taxi rank in the month of May 2013 during a community outreach campaign organized by the District Department of Health targeted at taxi ranks in Johannesburg. The measures used for data collection 
were categorized into sociodemographic characteristics, history of testing, and barriers to testing. Sociodemographic characteristics included participant age (under 15, 15-19, 20-24, 25-35, and older than 35 years), marital status (single, married, divorced, cohabitating), occupation (formally employed, self-employed, not employed, including students), education (below high school, high school, and post high school qualifications, including certificate, diploma, and degrees) distance to the nearest clinic (0-10, 11-20, 21-30, and more than $30 \mathrm{~km}$ ), place of residence (inner city, outside inner city), and place of work (inner city, outside inner city). The previous place where the last HIV test was done was categorized as never tested, clinic, hospital, mobile outreach, and home. During univariate and multivariate analysis, clinic and hospital as well as mobile outreach and home were combined. Payment for HCT services included all participants who answered yes to ever paying a fee for any HCT services they received. Sexual partners were assessed over 3 months, where participants were required to choose if they had none, one, or more than one sexual partner in the previous 3 months.

Potential barriers were identified by asking the study participants about the barriers to HIV testing. In this study, the following responses were used to generate an unordered outcome variable: paying for HCT services, negative attitude of health workers, influence of sexual partner, and stigma and discrimination. This was achieved by selecting the participants who answered yes to each question. Another question was asked to establish whether the participant's perceived risk of becoming HIV-infected was high or low. Geographic data were obtained freely from Internet sources. Quantum GIS was used to produce a map showing the location of the study area in relation to the city of Johannesburg (Figure 1).

\section{Statistical analysis}

The data analysis was performed using Stata version 12 (StataCorp, College Station, TX, USA). Missing observations in the outcome variable were excluded from analysis. Frequency distributions were used to describe categorical variables, as seen in Table 1. Five potential barriers were selected, ie, low perceived risk of HIV, paying for HCT services, poor health workers' attitudes, lack of partner support, and stigma and discrimination. These were used in five different logistic regression models to determine which correlates were associated with each of the potential barriers to HIV testing.

Bivariate logistic regression analysis was performed to assess the association between the selected covariates and
Table I Distribution of baseline characteristics of study participants $(n=I, \mid 46)$

\begin{tabular}{|c|c|c|}
\hline Factor & $\mathbf{n}$ & $\%$ \\
\hline \multicolumn{3}{|l|}{ Sex } \\
\hline Female & 579 & 50.5 \\
\hline Male & 567 & 49.5 \\
\hline \multicolumn{3}{|l|}{ Age group, years } \\
\hline$<15$ & 18 & 1.6 \\
\hline $15-19$ & 87 & 7.6 \\
\hline $20-24$ & 261 & 22.8 \\
\hline $25-35$ & 483 & 42.2 \\
\hline$>35$ & 297 & 25.9 \\
\hline \multicolumn{3}{|l|}{ Marital status } \\
\hline Single & 801 & 69.9 \\
\hline Cohabiting & 18 & 1.6 \\
\hline Married & 303 & 26.1 \\
\hline Divorced & 24 & 2.1 \\
\hline \multicolumn{3}{|l|}{ Employment status } \\
\hline Unemployed & 474 & 41.4 \\
\hline Self-employed & 312 & 27.2 \\
\hline Employed & 360 & 31.4 \\
\hline \multicolumn{3}{|l|}{ Education level } \\
\hline Below matriculation & 324 & 28.3 \\
\hline Matriculation & 198 & 43.5 \\
\hline Post matriculation & 324 & 28.3 \\
\hline \multicolumn{3}{|c|}{ Distance to nearest clinic, km } \\
\hline $0-10$ & 792 & 69.1 \\
\hline $\mathrm{II}-20$ & 180 & 15.7 \\
\hline $21-30$ & 66 & 5.8 \\
\hline$>30$ & 108 & 9.4 \\
\hline \multicolumn{3}{|l|}{ Place of residence } \\
\hline Inner city & 657 & 57.3 \\
\hline Outside inner city & 489 & 42.7 \\
\hline \multicolumn{3}{|c|}{ Area of employment } \\
\hline Inner city & 705 & 61.5 \\
\hline Outside inner city & 441 & 38.5 \\
\hline \multicolumn{3}{|c|}{ Place of previous HIV test* } \\
\hline Clinic & 696 & 60.7 \\
\hline Hospital & 165 & 14.4 \\
\hline Mobile outreach & 126 & 11.0 \\
\hline Home & 6 & 0.5 \\
\hline \multicolumn{3}{|l|}{ Sexual partners } \\
\hline None & 108 & 9.4 \\
\hline One & 681 & 59.4 \\
\hline More than one & 357 & 31.2 \\
\hline \multicolumn{3}{|c|}{ Payment for HCT services } \\
\hline No & $\mathrm{I}, 005$ & 87.7 \\
\hline Yes & $|4|$ & 12.3 \\
\hline \multicolumn{3}{|c|}{ Perceived risk of HIV } \\
\hline High & 552 & 48.2 \\
\hline Low & 594 & 51.8 \\
\hline
\end{tabular}

potential barriers to HCT. Multivariate logistics regression analysis using the backward elimination method was employed. Variables with a $P$-value $\leq 0.05$ on bivariate analysis were included into the multivariate logistic regression model. All factors with $P \leq 0.05$ were considered 
significant independent barriers to HCT, as shown in Table 2. The odds ratios (ORs) and corresponding 95\% confidence intervals (CIs) for potential barriers were obtained from regression analyses. Variables included in the analyses were: female sex, age 15-19 years, cohabiting, formally employed, below matriculation, more than $30 \mathrm{~km}$, and residence and employment in the inner city.

\section{Ethical approval}

Ethical approval for this study was obtained from the Monash University research ethics committee (approval CF14/2721-2014001511). Approval to use secondary data for this study was obtained from CARe, a nongovernmental organization. CARe provides mobile health services to communities and works in close collaboration with Monash University, South Africa. Verbal informed consent was obtained from individual participants at the time of primary data collection. No participant identifiers were collected.

\section{Results \\ Descriptive statistics}

The 1,146 study participants included 567 (49.5\%) males and $579(50.5 \%)$ females, with the majority (42.2\%) being aged 25-35 years. A total of $61.5 \%$ reported working in the inner city, with $57.3 \%$ of the participants living in the inner city. Of these, $69.1 \%$ reported living near a health facility, and approximately $58.6 \%$ were either in formal employment (31.4\%) or self-employment (27.2\%). Approximately $87 \%$ of the study population reported being previously tested for HIV and 51.8\% had a low-risk perception of acquiring HIV. Sixty-one percent reported testing for HIV less than a year earlier at clinic HIV testing centers (Table 1).

\section{Bivariate analysis}

Results of the bivariate analysis are presented in Table 2. Factors such as age group, marital status, occupation (selfemployed, formally employed), educational level, distance to the nearest clinic $(>30 \mathrm{~km})$, area of employment/residence (outside inner city), and numbers of sexual partners $(\geq 1)$. Factors that were significantly associated $(P<0.05)$ with potential barriers to HCT were further analyzed in multivariate logistic regression models.

\section{Multivariate regression analysis}

The results of the logistic regression analysis are presented in Table 2. Significant factors are outlined below.

\section{Low perceived risk of HIV}

Participants who were married were 1.5 times (OR 1.49; 95\% CI 1.13-1.96) more likely to report a low-risk perception of HIV as a barrier to HCT when compared with single participants. Participants with a high school qualification or above were less likely to report a low-risk perception of HIV as a potential barrier to HCT: high school (OR 0.67; 95\% CI 0.50-0.89) and post high school (OR 0.46; 95\% CI 0.33-0.63). Those who indicated hospitals as their previous place of HCT were less likely to mention a low-risk perception of HIV as a barrier to HCT (OR 0.73; 95\% CI 0.55-0.97).

\section{Lack of partner support}

Employed participants were less likely to cite lack of partner support as the reason for not testing for HIV compared with unemployed individuals: self-employed (OR $0.40 ; 95 \%$ CI $0.25-0.65$ ) and formally employed (OR 0.62; 95\% CI 0.41-0.95). Study participants living more than $30 \mathrm{~km}$ from a health facility were less likely to report lack of partner support as a potential barrier to HIV testing compared with those living less than $10 \mathrm{~km}$ from a health facility: $>30 \mathrm{~km}$ (OR 0.08; 95\% CI 0.02-2.66). Living outside the inner city increased the chances of reporting lack of partner support as a potential obstacle to HIV testing by 1.94 times compared with those living in the inner city (OR 1.94; 95\% CI 1.34-2.80). An increase in the number of sexual partners from one to two increased the likelihood of citing lack of partner support as a reason for not having an HIV test when compared with those who reported no sexual partners: one partner (OR 4.76; 95\% CI 1.45-15.69), more than one partner (OR 7.77; $95 \%$ CI 2.32-25.98).

\section{Stigma and discrimination}

A distance of more than $20 \mathrm{~km}$ from the nearest clinic increased the likelihood of citing stigma and discrimination as a barrier to HIV testing compared with a distance of less than $10 \mathrm{~km}$ : 21-30 km (OR 2.58; 95\% CI 1.48-4.49) and more than $30 \mathrm{~km}$ (OR 3.40; 95\% CI 2.20-5.25). An increase in the number of sexual partners reduced the likelihood of citing stigma and discrimination as a reason for not having an HIV test compared with those with no sexual partners: more than one partner (OR 0.73; 95\% CI 0.53-0.99).

\section{Poor health workers' attitudes}

An increase in educational level was significantly associated with reporting health workers' attitudes as a barrier to HIV 


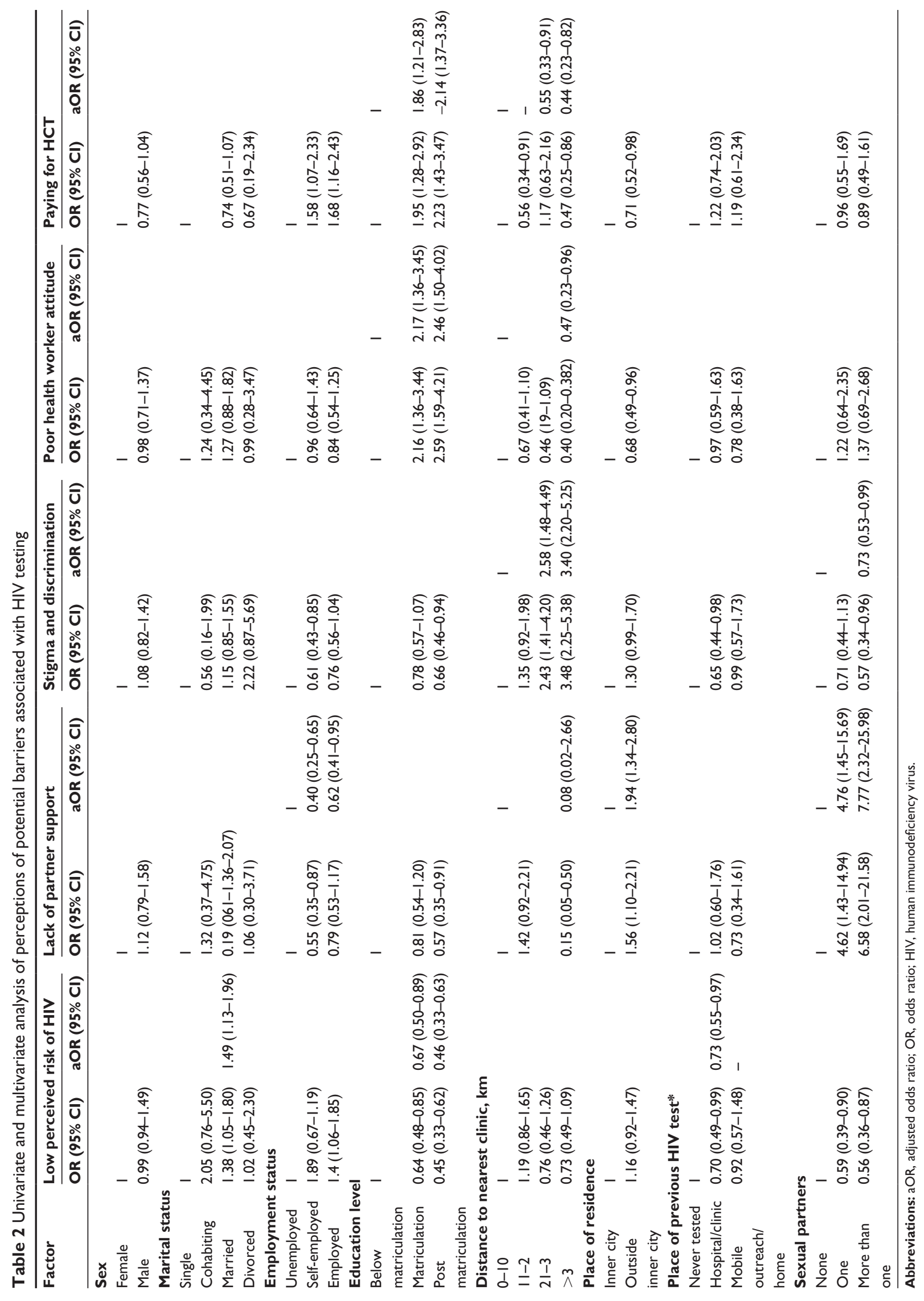


testing compared with no high school education: high school (OR 2.17; 95\% CI 1.36-3.45) and post high school (OR 2.46; $95 \%$ CI 1.50-4.02). In this study, long distance from a health facility had a protective effect against non-testing as a result of poor health workers' attitudes when compared with those who lived less than $10 \mathrm{~km}$ from the nearest clinic/health facility: $>30 \mathrm{~km}$ (OR 0.47; 95\% CI 0.23-0.96).

\section{Paying for HCT}

Participants with a high school qualification or above were more likely to report paying for HCT services as a reason for not testing compared with those without a high school education: high school (OR 1.86; 95\% CI 1.21-2.83) and post high school (OR 2.14; 95\% CI 1.37-3.36). Similar to poor health workers' attitude, the greater the distance to the nearest clinic, the less likely it was that the subject would report paying for HCT services as a reason for not having an HIV test: $21-30 \mathrm{~km}($ OR $0.55 ; 95 \%$ CI $0.33-0.91)$ and $>30 \mathrm{~km}$ (OR 0.44; 95\% CI 0.23-0.82).

\section{Discussion}

This study found the following factors to be significantly associated with potential barriers to HIV testing: marital status, occupation, educational level, area of employment and residence, distance to the nearest clinic, and numbers of sexual partners. HCT services provide a platform via which millions of people can get to know their HIV status and become linked to care and treatment should they test positive for the virus. Despite the crucial role that HCT plays in control of HIV transmission and access to treatment, approximately $50 \%$ of people living with the virus do not know their HIV status, according to the 2012 UNAIDS report. Several factors contribute to this, and are the barriers that prevent people attending for HCT. In light of this, we investigated the barriers to accessing HCT services in the commuter population within the Johannesburg metropolitan health district. To our knowledge, this is the first study to assess barriers to HIV testing among the mobile population in Johannesburg.

We found that approximately $87 \%$ of the study population had tested for HIV and more than half of them perceived their risk of contracting the virus as low. In another venue intercept study that was conducted in Cape Town, $47 \%$ of participants had tested for HIV and the risk of exposure to HIV was high and comparable among people tested and not tested. ${ }^{12}$ Previous studies also point to low perceived risk as a problematic barrier to HIV testing. When one does not perceive that one engages in high-risk behaviors, one does not have the need to test for HIV. However, one's self-perceived risk is often inaccurate. ${ }^{13}$ Furthermore, perception of lower risk correlates with higher rates of HIV infection. ${ }^{14}$

Participants with high school and post high school qualifications were less likely to report stigma and discrimination as a factor hindering HIV testing, although education was not significant in multivariate analysis. HIV and AIDS-related stigma has been cited as a barrier to HIV testing despite the presence of knowledge about HIV transmission. ${ }^{12}$ Shisana et al found that HIV/AIDS-related stigma in South Africa has actually been decreasing since 2002. However, in a recent study published in 2012, HIVrelated stigma was shown to be a prominent barrier to testing in South Africa. ${ }^{10}$ Lessening of stigma and discrimination is a key aspect in increasing the uptake of HIV testing ${ }^{23}$ in the population.

Poor health worker attitude was identified as a significant factor influencing not testing among participants. Participants with high school education and above were more likely to report poor health worker attitude as a factor hindering testing for HIV. Some studies have reflected the need to provide training of health workers so as to improve health worker attitude, which may lead to an increase in uptake of HIV testing. ${ }^{24,25}$

Participants who lived furthest from the health facility were less likely to report poor health worker attitude, paying for HCT services, and lack of partner support as barriers to HIV testing. This is an indication that distance is no longer a significant aspect, as health services may be accessed. Participants who lived outside the inner city were more likely to indicate lack of partner support as a potential barrier to HCT compared with those living in the inner city.

\section{Limitations of the study}

The venue-based intercept survey design of this study is a weakness; a multi-venue taxi rank sampling strategy could have been employed to obtain a cross-section of inhabitants of the city of Johannesburg. The refusal rates with recruitment of respondents at the taxi rank were also not captured. Indepth interviews could have given the study some depth in understanding the barriers to uptake of HCT, and this was not done. The study was conducted in 1 day, whereas conducting it over a longer period might have given more varied findings. The use of personal tablets as data collection tools might have prevented potential technophobic or elderly participants from answering the survey. However, support was provided for those who needed assistance from the field 
staff. Further, the data obtained were self-reported, which may result in social desirability bias. Because of these limitations, caution should be exercised before generalizing the study results to other areas and population groups with similar characteristics.

\section{Conclusion}

Marital status, occupation, educational level, area of employment and residence, distance to the nearest clinic, and numbers of sexual partners were factors that were significantly associated with barriers to HIV testing in the study population. For future interventions, health services provision, must take cognizance of these factors which are associated with Barriers to HIV testing. Health education plays an important role and the need to design age-specific messages is important in the design of programs. Meanwhile, a high level of stigma and discrimination with regard to HIV testing was observed in this population. More programs need to be designed so as to address stigma, particularly among single people. Multiple and/or concurrent sexual partners are among the key drivers of HIV infection; hence, dealing with this issue is of vital importance to decrease the stigma associated with accessing HCT services. There is a need to identify strategies to address barriers to testing in the commuter population and institute effective public health interventions to increase uptake of HCT in this population.

\section{Acknowledgment}

Funding for the field staff in this study was provided by the Independent Development Trust and Right to Care. The funder had no role in the study design, data collection and analysis, decision to publish, or preparation of the manuscript.

\section{Disclosure}

The authors report no conflicts of interest in this work.

\section{References}

1. Statistics South Africa. Mid-year population estimates: Statistical release. 2013. Available from: http://www.statssa.gov.za.

2. Suthar AB, Ford N, Bachanas PJ, et al. Towards universal voluntary HIV testing and counselling: a systematic review and meta-analysis of community-based approaches. PLoS Med. 2013;10:e1001496.

3. Weinreb A, Stecklov G. Social inequality and HIV-testing: comparing home- and clinic-based testing in rural Malawi. Demogr Res. 2009;21:627-646.

4. Mutale W, Michelo C, Jürgensen M, Fylkesnes K. Home-based voluntary HIV counselling and testing found highly acceptable and to reduce inequalities. BMC Public Health. 2010;10:347.

5. Cherutich P, Bunnell R, Mermin J. HIV testing: current practice and future directions. Curr HIV/AIDS Rep. 2013;10:134-141.

6. Shisana O, Rehle T, Simbayi LC, et al. South African national HIV prevalence, incidence, behaviour and communication survey, 2008: a turning tide among teenagers? 2009. Available from: http://www.health-e.org. za/wp-content/uploads/2013/05/2966e129fc39e07486250fd47fcc266e. pdf. Accessed November 1, 2014.
7. World Health Organization. HIV testing and counseling. 2013. Available from: http://www.who.int/hiv/topics/vct/en/. Accessed November 1, 2014.

8. Kamb ML, Fishbein M, Douglas JM, et al. Efficacy of risk-reduction counseling to prevent human immunodeficiency virus and sexually transmitted diseases: a randomized controlled trial. Project RESPECT Study Group. JAMA. 1998;280:1161-1167.

9. Department of Health. Outline of the national HIV Counselling and Testing (HCT) campaign. 2010.

10. Pitpitan EV, Kalichman SC, Eaton LA, et al. AIDS-related stigma, HIV testing, and transmission risk among patrons of informal drinking places in Cape Town, South Africa. Ann Behav Med. 2012;43: 362-371.

11. Doherty TM, McCoy D, Donohue S. Health system constraints to optimal coverage of the prevention of mother-to-child HIV transmission programme in South Africa: lessons from the implementation of the national pilot programme. Afr Health Sci. 2005;5:213-218.

12. Kalichman SC, Simbayi LC. HIV testing attitudes, AIDS stigma, and voluntary HIV counselling and testing in a black township in Cape Town, South Africa. Sex Transm Infect. 2003;79:442-447.

13. Johnston L, O'Bra H, Chopra M, et al. The associations of voluntary counseling and testing acceptance and the perceived likelihood of being HIV-infected among men with multiple sex partners in a South African township. AIDS Behav. 2010;14:922-931.

14. Shisana O, Rehle T, Simbayi L. South African national HIV prevalence, HIV incidence, behavior and communication survey, 2005. Cape Town, South Africa .... 2005.

15. Ziraba AK, Madise NJ, Kimani JK, et al. Determinants for HIV testing and counselling in Nairobi urban informal settlements. BMC Public Health. 2011;11:663.

16. Statistics South Africa. Census 2011 Statistical Release. 2012.

17. Shtarkshall R, Soskolne V. Migrant Populations and HIV/AIDS: The Development and Implementation of Programmes Theory, Methodology and Practice. Geneva, Switzerland: United Nations Educational, Scientific and Cultural Organization/Joint United Nations Programme on HIV/AIDS; 2000.

18. Venkatesh KK, Madiba P, De Bruyn G, Lurie MN, Coates TJ, Gray GE. Who gets tested for HIV in a South African urban township? Implications for test and treat and gender-based prevention interventions. J Acquir Immune Defic Syndr. 2011;56:151-165.

19. Kigozi NG, Heunis JC, Wouters E, van den Berg HS. Tuberculosis patients' reasons for, and suggestions to address non-uptake of HIV testing: a cross-sectional study in the Free State Province, South Africa. BMC Health Serv Res. 2011;11:110.

20. Muloongo K, Tshuma N, Chimoyi L, Setswe G, Sarfo B, Nyasulu P. Factors contributing to home-based acceptability of rapid testing for HIV infection among the inner city commuter population in Johannesburg, South Africa. Trans R Soc Trop Med Hyg. 2014;108:632-638.

21. COJ. city of Johannesburg - Taxis. 2013.

22. Ayala A, Geurts E, Ahmad P, et al. Urbanising Africa: the city centre revisited. 2010;26:1-80.

23. Stangl AL, Lloyd JK, Brady LM, Holland CE, Baral S. Review article A systematic review of interventions to reduce HIV-related stigma and discrimination from 2002 to 2013 : how far have we come? J Int AIDS Soc. 2013;16(3 Suppl 2):18734.

24. European Centre for Disease Prevention and Control. HIV testing: Increasing uptake effectiveness in the European Union. Stockholm, Sweden: European Centre for Disease Prevention and Control; 2010. Available from: http://ecdc.europa.eu/en/publications/Publications/101129_GUI_HIV_testing.pdf. Accessed November 1, 2014.

25. Musheke M, Ntalasha H, Gari S, et al. A systematic review of qualitative findings on factors enabling and deterring uptake of HIV testing in Sub-Saharan Africa. BMC Public Health. 2013;13:220. 
HIV/AIDS - Research and Palliative Care

Dovepress

\section{Publish your work in this journal}

HIV/AIDS - Research and Palliative Care is an international, peerreviewed open-access journal focusing on advances in research in HIV, its clinical progression and management options including antivira treatment, palliative care and public healthcare policies to control viral spread. The journal welcomes original research, basic science, clinical \& epidemiological studies, reviews \& evaluations, expert opinion \& commentary, case reports \& extended reports. The manuscript management system is completely online and includes a very quick and fair peer-review system. Visit http://www.dovepress.com/ testimonials.php to read real quotes from published authors.

Submit your manuscript here: http://www.dovepress.com/hivaids---research-and-palliative-care-journal 